\title{
OPE of Green functions in the odd sector of QCD
}

\author{
T. Kadavý ${ }^{1, a}$, K. Kampf ${ }^{1}$, and J. Novotný ${ }^{1}$ \\ ${ }^{1}$ Charles University, Faculty of Mathematics and Physics, Prague, Czech Republic
}

\begin{abstract}
A review of familiar results of the three-point Green functions of currents in the odd-intrinsic parity sector of QCD is presented. Such Green functions include very well-known examples of $V V P, V A S$ or $A A P$ correlators. We also present new results for $V V A$ and $A A A$ Green functions that have not yet been studied extensively in the literature before, more importantly with a phenomenological study and a discussion of the highenergy behaviour and its relation to the QCD condensates.
\end{abstract}

\section{Introduction}

The Green functions are defined as the vacuum expectation values of the time ordered products of the composite operators $O_{i}\left(x_{i}\right)$. In the momentum representation, the standard definition of the three-point Green functions reads

$$
\int \mathrm{d}^{4} x_{1} \mathrm{~d}^{4} x_{2} e^{i\left(p_{1} x_{1}+p_{2} x_{2}\right)}\left\langle 0\left|\mathrm{~T}\left[O_{1}\left(x_{1}\right) O_{2}\left(x_{2}\right) O_{3}(0)\right]\right| 0\right\rangle .
$$

In our case, the composite operators $O_{i}\left(x_{i}\right)$ stand either for chiral vector $V^{\mu, a}=\bar{q} \gamma^{\mu} T^{a} q$ and axialvector $A^{\mu, a}=\bar{q} \gamma^{\mu} \gamma_{5} T^{a} q$ currents, or scalar $S^{a}=\bar{q} T^{a} q$ and pseudoscalar $P^{a}=i \bar{q} \gamma_{5} T^{a} q$ densities, where $q$ stands for the quark triplet that contains the light quarks $(u, d, s), T^{a}$ is a SU(3) generator, $T^{a}=\lambda^{a} / 2$, and $a=1 \ldots 8$ are the group indices. Obviously, all the group and Lorentz indices in the definition (1) above are suppresed for simplicity.

As we mentioned above, there exist only five nontrivial three-point Green functions in the odd parity sector of QCD. The nonvanishing combinations of currents and densities are the following: $V V P, V A S, A A P, V V A$ and $A A A$. There are two regimes where the QCD dynamics of the current correlators is well understood.

The first one is that of low external momenta where the dynamics is governed by the approximate chiral symmetry of low-energy QCD. In detail, the spontaneous breaking of the chiral $\mathrm{SU}(3)_{L} \times \mathrm{SU}(3)_{R}$ symmetry down to $\mathrm{SU}(3)_{V}$ in QCD leads to the presence of Goldstone bosons. By identifying them with the octet of pseudoscalar mesons $(\pi, K, \eta)$ as the lightest hadronic observable states, we can construct chiral perturbation theory $(\chi \mathrm{PT})$ as an effective theory of QCD for energies $E \leq M_{\rho}$, where $M_{\rho}$ is the mass of $\rho$ meson [1], [2], [3]. For energies $M_{\rho} \leq E \leq 2 \mathrm{GeV}$ the effective theory is resonance chiral theory $(\mathrm{R} \chi \mathrm{T})[4]$. Such theory increases the number of degrees of freedom of $\chi$ PT by including massive U(3) multiplets of vector $V\left(1^{--}\right)$, axial-vector $A\left(1^{++}\right)$, scalar $S\left(0^{++}\right)$and pseudoscalar $P\left(0^{-+}\right)$ resonances.

\footnotetext{
ae-mail: kadavy@ipnp.troja.mff.cuni.cz
} 
The second regime corresponds to the high energies where the asymptotic freedom allows us to use the perturbative approach in terms of the strong coupling $\alpha_{s}$ and where the asymptotics of the correlators for large euclidean momenta is given by the operator product expansion (OPE). Within the OPE framework, short-distance behaviour of the Green functions can be studied. For example, let us consider a product of two operators $A(x)$ and $B(y)$. Then, the OPE is equivalent to an assumption that at large external momentum $p$, the two-point Green function of the operators above can be rewritten in the form

$$
i \int \mathrm{d}^{4} x e^{i p x}\langle 0|\mathrm{~T}[A(x) B(y)]| 0\rangle=\sum_{n} C_{n}^{A B}\left(p^{2}\right)\left\langle 0\left|O_{n}\right| 0\right\rangle,
$$

where $\left\langle 0\left|O_{n}\right| 0\right\rangle$ are the vacuum averages of composite gauge-invariant local operators, made of quark and gluon fields. Note that only the spin-zero operators contribute to the vacuum expectation value. Such vacuum averages are usually called the QCD condensates. Considering the dimension of such condensates to be $D \leq 6$, their complete set in massless theory is as follows:

$$
\begin{aligned}
\text { the unit operator: } & O_{0}=1, \\
\text { quark condensate: } & O_{3}=\langle 0|\bar{q} q| 0\rangle, \\
\text { gluon condensate: } & O_{4}=\left\langle 0\left|G_{\mu \nu} G^{\mu v}\right| 0\right\rangle, \\
\text { quark-gluon condensate: } & O_{5}=\left\langle 0\left|\bar{q} \sigma_{\mu \nu} G^{\mu v} q\right| 0\right\rangle, \\
\text { four-quark condensate: } & O_{6}^{q}=\langle 0|\bar{q} \Gamma q \bar{q} \Gamma q| 0\rangle, \\
\text { three-gluon condensate: } & O_{6}^{G}=\left\langle 0\left|G_{\mu \nu}^{a} G_{v \sigma}^{b} G_{\sigma \mu}^{c}\right| 0\right\rangle f^{a b c},
\end{aligned}
$$

where $\Gamma$ stands for any combination of the $4 \times 4$ matrices of a set $\left\{1, \gamma_{5}, \gamma^{\mu}, \gamma_{5} \gamma^{\mu}, \sigma^{\mu \nu}\right\}$ together with the $3 \times 3$ matrices $\left\{1, T^{a}\right\}, a=1 \ldots 8$, that is Lorentz invariant. In the following sections we will see that such QCD condensates play an important role in evaluation of the OPE of the corresponding Green functions.

It seems that the three-point OPE has been known only for three out of five nontrivial Green functions in the odd-intrinsic parity sector of QCD, specifically for $V V P, V A S$ and $A A P$. Therefore, the study of the OPE for $V V A$ and $A A A$ correlators is thus given by our contribution.

\subsection{Notation}

In this article, we consider all momenta $p, q, r$ as ingoing to the vertices, i.e. the law of momentum conservation can be written in the form

$$
p+q+r=0 .
$$

Also, a notation for the contractions of Levi-Civita tensor with the four-momenta is defined as

$$
\varepsilon_{\mu \nu \alpha(p)} \equiv \varepsilon_{\mu \nu \alpha \beta} p^{\beta}, \quad \varepsilon_{\mu v(p)(q)} \equiv \varepsilon_{\mu \nu \alpha \beta} p^{\alpha} q^{\beta} .
$$

\section{Review of familiar results}

As we have already mentioned, the Green functions that have been already studied extensively in the literature are $V V P, V A S$ and $A A P$. All three of them can be written in the simple forms

$$
\begin{aligned}
& \left(\Pi_{V V P}(p, q ; r)\right)_{\mu v}^{a b c}=\Pi_{V V P}\left(p^{2}, q^{2} ; r^{2}\right) d^{a b c} \varepsilon_{\mu v(p)(q)}, \\
& \left(\Pi_{V A S}(p, q ; r)\right)_{\mu v}^{a b c}=\Pi_{V A S}\left(p^{2}, q^{2} ; r^{2}\right) f^{a b c} \varepsilon_{\mu v(p)(q)}, \\
& \left(\Pi_{A A P}(p, q ; r)\right)_{\mu v}^{a b c}=\Pi_{A A P}\left(p^{2}, q^{2} ; r^{2}\right) d^{a b c} \varepsilon_{\mu \nu(p)(q)} .
\end{aligned}
$$


The high-energy behaviour within the OPE framework can be written down as follows. If we consider all individual external momenta of all the three currents or densities to be high, it is easy to realize that the leading order contribution corresponds to two contractions of the quark fields contained in the respective currents and densities. The three contractions are already equal to zero, whilst one and none contractions are subleading. Then, the LO contribution to the OPE is generated only by the quark condensate and it can be illustrated as (the crossed circle stands either for the current or the density of respective type)

$$
\langle 0|\rightarrow \bigotimes \rightarrow \bigotimes \rightarrow-\otimes \rightarrow| 0\rangle \neq 0 .
$$

The explicit calculations lead to the results [5], [6]:

$$
\begin{aligned}
& \Pi_{V V P}\left((\lambda p)^{2},(\lambda q)^{2} ;(\lambda r)^{2}\right)=\frac{B_{0} F^{2}}{2 \lambda^{4}} \frac{p^{2}+q^{2}+r^{2}}{p^{2} q^{2} r^{2}}+O\left(\frac{1}{\lambda^{6}}\right), \\
& \Pi_{V A S}\left((\lambda p)^{2},(\lambda q)^{2} ;(\lambda r)^{2}\right)=\frac{B_{0} F^{2}}{2 \lambda^{4}} \frac{p^{2}-q^{2}-r^{2}}{p^{2} q^{2} r^{2}}+O\left(\frac{1}{\lambda^{6}}\right), \\
& \Pi_{A A P}\left((\lambda p)^{2},(\lambda q)^{2} ;(\lambda r)^{2}\right)=\frac{B_{0} F^{2}}{2 \lambda^{4}} \frac{p^{2}+q^{2}-r^{2}}{p^{2} q^{2} r^{2}}+O\left(\frac{1}{\lambda^{6}}\right) .
\end{aligned}
$$

A detailed explanation of calculations within the OPE framework can be found in [7], [8].

So far, our discussion was rather of a mathematical nature. Hence, let us introduce the tools we use in our analysis. Dispersion representation of the Green functions which are order parameters of the chiral symmetry breaking enables us to make use of an information on the asymptotics both at the low and high energies and allows us to relate the unknown low energy constants (LECs) to the properties of the corresponding spectral functions in terms of the chiral sum rules. These are usually assumed to be saturated by the resonant states at low energies. This assumption connects the LECs to the phenomenology of resonances in the intermediate energy region, i.e. for energies $M_{\rho} \leq E \leq 2 \mathrm{GeV}$. The next-to-leading order resonance Lagrangian, relevant in the odd-intrinsic parity sector of QCD, was formulated for the first time in [9] and is of the form

$$
\mathcal{L}_{R}^{(6)}=\sum_{X} \sum_{i} \kappa_{i}^{X} \widehat{O}_{i \mu \nu \alpha \beta}^{X} \varepsilon^{\mu \nu \alpha \beta},
$$

where $X$ stands for the single-resonance fields $V, A, S, P$, double-resonance fields $V V, A A, S A, S V$, $V A, P A, P V$ and triple-resonance fields $V V P, V A S, A A P$. This Lagrangian contains 67 operators $\widehat{O}_{i \mu \nu \alpha \beta}^{X}$ in total and the same number of corresponding unknown coupling constants $\kappa_{i}^{X}$. We try to determine the couplings using the following constraints: high-energy behavior (OPE), Brodsky-Lepage behavior of the transition formfactor, matching calculations in $\mathrm{R} \chi \mathrm{T}$ with $\chi \mathrm{PT}$ or suitable inputs from experiments.

\section{OPE for $V V A$ Green function with two large momenta}

Before we make the same attempt for the remaining $V V A$ and $A A A$ correlators, let us make use of an already known result of the two-point OPE for the $V V A$, where only two external momenta are large. In the standard definition of the $V V A$ Green function, i.e. the correlator of two vector and one axial-vector current, one can factorize the symmetric tensor that comes from the traces over flavor space and the Lorentz part, which is restricted by the Ward identities into four terms, i.e.

$$
\left(\Pi_{V V A}(p, q ; r)\right)_{\mu \nu \rho}^{a b c}=d^{a b c}\left[w_{L} \varepsilon_{\mu \nu(p)(q)} r_{\rho}+w_{T}^{(1)} \Pi_{\mu \nu \rho}^{(1)}+w_{T}^{(2)} \Pi_{\mu \nu \rho}^{(2)}+w_{T}^{(3)} \Pi_{\mu \nu \rho}^{(3)}\right]
$$


where $\Pi_{\mu \nu \rho}^{(i)}(i=1,2,3)$ are transversal tensors defined in [10]. The longitudinal part proportional to form factor $w_{L}$ is fixed by the anomaly, whilst $w_{T}^{(i)}$ are transversal form factors. In order to identify the unknown form factors $w_{L}, w_{T}^{(i)}$ we have calculated all possible Feynman diagrams at tree level that contribute to the $V V A$ Green function and that are generated by the resonance Lagrangian (18). Our calculations [11] lead to the results

$$
\begin{aligned}
w_{L} & =\frac{N_{c}}{8 \pi^{2} r^{2}}, \\
w_{T}^{(1)} & =-\frac{2 \sqrt{2} F_{V}\left[\kappa_{17}^{V}\left(p^{2}+q^{2}-2 M_{V}^{2}\right)-\sqrt{2} F_{V} \kappa_{3}^{V V}\right]}{\left(p^{2}-M_{V}^{2}\right)\left(q^{2}-M_{V}^{2}\right)}, \\
w_{T}^{(2)} & =-\frac{2 \sqrt{2} F_{V}\left(p^{2}-q^{2}\right)\left(2 \kappa_{12}^{V}+\kappa_{16}^{V}-\kappa_{17}^{V}\right)}{\left(p^{2}-M_{V}^{2}\right)\left(q^{2}-M_{V}^{2}\right)}, \\
w_{T}^{(3)} & =\frac{2 \sqrt{2} F_{V}\left(p^{2}-q^{2}\right)}{\left(p^{2}-M_{V}^{2}\right)\left(q^{2}-M_{V}^{2}\right)}\left(2 \kappa_{11}^{V}+2 \kappa_{12}^{V}-\kappa_{17}^{V}-\frac{\sqrt{2} F_{A} \kappa_{5}^{V A}}{r^{2}-M_{A}^{2}}\right) .
\end{aligned}
$$

A comment is in order here. Unlike in our case of the $V V A$ correlator, where we have vertices with two resonances at most, one could also have large- $N_{c}$ diagrams contributing to the three-point Green functions with three resonances. This may seem to be the case, considering the expression (23), where one can find a term made of three resonance propagators. However, this observation is just a coincidence due to algebraical manipulations. One can rewrite the term $\left(p^{2}-q^{2}\right)$ in front of the brackets in (23) simply as $\left(p^{2}-M_{V}^{2}\right)-\left(q^{2}-M_{V}^{2}\right)$ which leads to the original structure of $w_{T}^{(3)}$ consisted of two resonance propagators, as one should expect from the form of the operator contributing with the coupling constant $\kappa_{5}^{V A}$ (see [11] for details).

Let us make a use of some known relations concerning the OPE for the VVA Green function, when only two out of three momenta are large. Then, we construct the formactor [10]

$$
w_{T}\left(Q^{2}\right)=-16 \pi^{2}\left[w_{T}^{(1)}\left(-Q^{2}, 0,-Q^{2}\right)+w_{T}^{(3)}\left(-Q^{2}, 0,-Q^{2}\right)\right]
$$

Be aware of the different notation here and in [10], although the definition (24) is defined here in order to be the same. An important fact is that the result for 24) up to $O\left(1 / Q^{8}\right)$ was obtained from the OPE framework in AdS/QCD [12, 13], in which the outcome reads

$$
w_{T}\left(Q^{2}\right)=\frac{N_{c}}{Q^{2}}+\frac{128 \pi^{3} \alpha_{s} \chi\langle\bar{q} q\rangle^{2}}{9 Q^{6}}+O\left(\frac{1}{Q^{8}}\right),
$$

where $\chi$ is the magnetic susceptibility of the quark condensate - a detailed introduction and definition of this quantity can be found in [13]. Using [21] and [23], we simply obtain the formfactor [24] in the form

$$
w_{T}\left(Q^{2}\right)=\frac{N_{c}}{M_{V}^{2}}+\frac{64 \pi^{2} F_{V}}{M_{V}^{2}\left(Q^{2}+M_{V}^{2}\right)}\left[Q^{2}\left(\sqrt{2}\left(\kappa_{11}^{V}+\kappa_{12}^{V}\right)+\frac{F_{A} \kappa_{5}^{V A}}{Q^{2}+M_{A}^{2}}\right)-F_{V} \kappa_{3}^{V V}\right],
$$

where we have already made a use of the known expression for the coupling constant $\kappa_{17}^{V}$ from the constraint $\kappa_{17}^{V}=-\frac{N_{c}}{64 \sqrt{2} \pi^{2} F_{V}}$ for $V V P$ Green function (see [9] for details). Now, we expand 26] into a series in terms of $Q^{2}$ up to $O\left(1 / Q^{8}\right)$ and compare the coefficients of the given order with 25]. This 
leads to the system of four equations, which can be easily solved:

$$
\begin{gathered}
\frac{N_{c}}{64 \pi^{2} F_{V}}+\sqrt{2}\left(\kappa_{11}^{V}+\kappa_{12}^{V}\right)=0, \\
\frac{F_{V} \kappa_{3}^{V V}-F_{A} \kappa_{5}^{V A}}{M_{V}^{2}}+\sqrt{2}\left(\kappa_{11}^{V}+\kappa_{12}^{V}\right)=-\frac{N_{c}}{64 \pi^{2} F_{V}}, \\
\frac{F_{V} \kappa_{3}^{V V}-F_{A} \kappa_{5}^{V A}}{M_{V}^{2}}+\sqrt{2}\left(\kappa_{11}^{V}+\kappa_{12}^{V}\right)-F_{A} \kappa_{5}^{V A} \frac{M_{A}^{2}}{M_{V}^{4}}=0, \\
\frac{F_{V} \kappa_{3}^{V V}-F_{A} \kappa_{5}^{V A}}{M_{V}^{2}}+\sqrt{2}\left(\kappa_{11}^{V}+\kappa_{12}^{V}\right)-F_{A} \kappa_{5}^{V A} \frac{M_{A}^{2}}{M_{V}^{4}}\left(1+\frac{M_{A}^{2}}{M_{V}^{2}}\right)=-\frac{2 \pi \alpha_{s} \chi\langle\bar{q} q\rangle^{2}}{9 F_{V} M_{V}^{4}} .
\end{gathered}
$$

From the first equation (27) we obtain the solution in the form (31). By using this solution in (28), we get a simple relation between the couplings $\kappa_{3}^{V V}$ and $\kappa_{5}^{V A}$ in the form $\kappa_{5}^{V A}=\frac{F_{V}}{F_{A}} \kappa_{3}^{V V}$. From (29) we eventually obtain a specific form for both of these couplings, (32) and (33). The results are summarized here:

$$
\begin{aligned}
\kappa_{11}^{V}+\kappa_{12}^{V} & =-\frac{N_{c}}{64 \sqrt{2} \pi^{2} F_{V}}, \\
\kappa_{3}^{V V} & =-\frac{N_{c} M_{V}^{4}}{64 \pi^{2} M_{A}^{2} F_{V}^{2}}, \\
\kappa_{5}^{V A} & =-\frac{N_{c} M_{V}^{4}}{64 \pi^{2} M_{A}^{2} F_{V} F_{A}} .
\end{aligned}
$$

Having known the constraints above, we can substitute them in (30), which on the left side of the equation gives an expression $-N_{c} M_{A}^{2} M_{V}^{2}$ which obviously can not be matched to the right side of the equation. Therefore, the system of equations (27)- (30) can not be solved in order to satisfy all the equations at once. Hence, the obtained constraints 31 - 33 need not to be taken seriously, but more likely as a close approximation to reality.

Also, using the constraint for $\kappa_{3}^{V V}$ from the OPE for $V V P$ Green function together with our result [32), we can extract relations for $\kappa_{2}^{V V}$ and $\kappa_{3}^{P V}$ (see [9] for details):

$$
\begin{aligned}
& \kappa_{2}^{V V}=\frac{F^{2}}{64 F_{V}^{2}}-\frac{N_{c} M_{V}^{4}}{512 \pi^{2} F_{V}^{2} M_{A}^{2}}, \\
& \kappa_{3}^{P V}=-\frac{F^{2}}{32 \sqrt{2} d_{m} F_{V}}\left[1+\frac{N_{c} M_{V}^{2}}{8 \pi^{2} F^{2}}\left(\frac{M_{V}^{2}}{M_{A}^{2}}-1\right)\right] .
\end{aligned}
$$

By the determination of (35) we can also obtain a relation for the deviation $\delta_{\mathrm{BL}}$ from the form of $\kappa_{3}^{P V}$ if we take the Brodsky-Lepage behaviour [9, 14, 15] of the $\mathcal{F}_{\pi^{0} \gamma \gamma}$ formfactor into account. Hence, the prediction is

$$
\delta_{\mathrm{BL}}=\frac{N_{c} M_{V}^{2}}{8 \pi^{2} F^{2}}\left(\frac{M_{V}^{2}}{M_{A}^{2}}-1\right)=-1.342 .
$$

Now, a discussion involving the parameter $\delta_{\mathrm{BL}}$ is in order. Knowing the value [36, , one can apply it to study a particular example that could be verified either by other theoretical consequences or experiments. In our case, a $\pi^{0} \gamma \gamma$ form factor is a suitable tool, because the experimentally measured 
object

$$
\mathcal{F}_{\pi^{0} \gamma \gamma}^{\mathrm{R} \chi \mathrm{T}}\left(0,-Q^{2} ; 0\right)=\frac{F}{3 M_{V}^{2}\left(Q^{2}+M_{V}^{2}\right)}\left(Q^{2} \delta_{\mathrm{BL}}-\frac{N_{c} M_{V}^{4}}{4 \pi^{2} F^{2}}\right),
$$

is sensitive to the value of $\delta_{\mathrm{BL}}$. The form factor (37) is depicted in figure 1 for our value $\delta_{\mathrm{BL}}=-1.342$ and for two other values, based on [9]. The figure also contains experimentally obtained values of $\mathcal{F}_{\pi^{0} \gamma \gamma}^{\mathrm{R} \chi \mathrm{T}}\left(0,-Q^{2} ; 0\right)$ from experiments BABAR [16], BELLE [17] and CLEO [18].

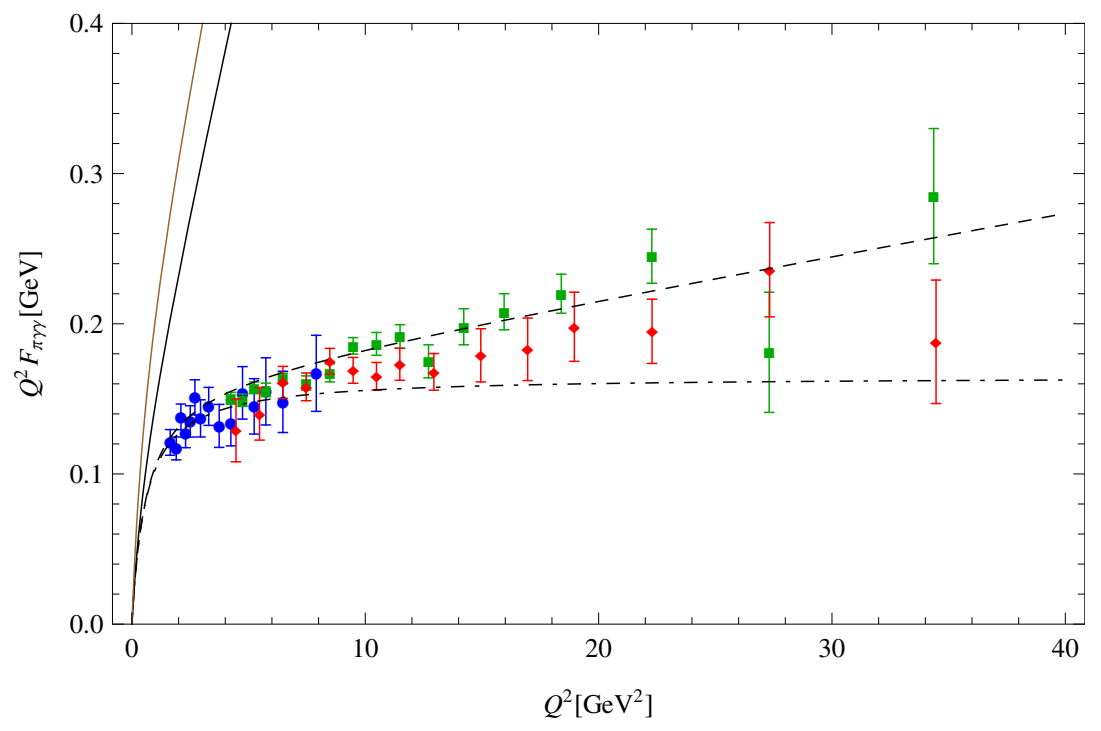

Figure 1. A plot of BABAR (green), BELLE (red) and CLEO (blue) data fitted with the form factor $\mathcal{F}_{\pi^{0} \gamma \gamma}^{\mathrm{RT}}\left(0,-Q^{2} ; 0\right)$ 37 using the modified Brodsky-Lepage condition. The full black line represents the form factor with $\delta_{\mathrm{BL}}=-1.342$, the dashed line corresponds to $\delta_{\mathrm{BL}}=-0.055$ (see [9]) and the dot-dashed line to $\delta_{\mathrm{BL}}=0$. The full brown line stands for the LMD form factor 38 .

We can clearly see that the form factor (37) does not agree with the experimental data. To get a full notion, one should discuss first if we even have a sufficiently consistent theoretical model to describe such behaviour. In other words, is it sufficient not to add any other resonance fields and still have an agreement with the experiments? Obviously, not. The reason is that our form factor (37) for the value $\delta_{\mathrm{BL}}=-1.342$ is very close to the behaviour of the form factor $\mathcal{F}^{\mathrm{LMD}}\left(p^{2}, q^{2} ; r^{2}\right)$ describing the lowest meson dominance (LMD) [19], [20]:

$$
\mathcal{F}^{\mathrm{LMD}}\left(0,-Q^{2} ; 0\right)=-\frac{N_{c}}{8 \pi^{2} F} \frac{M_{V}^{2}}{Q^{2}+M_{V}^{2}}\left(1+\frac{4 \pi^{2} F^{2}}{N_{c} M_{V}^{4}} Q^{2}\right) .
$$

This means that it is not obviously sufficient to take only the lightest resonances into account and one thus needs to add heavier fields in order to get closer to the experimantal data. To support our explanation, the LMD form factor 38 is also depicted in figure 1 . 


\section{OPE for $V V A$ and $A A A$ Green functions with three large momenta}

As we have seen above, the predicted value for $\delta_{\mathrm{BL}}$ is not consistent with the experimental data. Let us remind ourselves that such a prediction is based on the OPE with two large momenta. However, there is no physical argument that would prefer such a type of the OPE. Hence, one can try to calculate the OPE with all three momenta large and find out if the agreement between the results and experiment improves. However, such an approach is not that easy in the case of the $V V A$ and $A A A$ Green functions, because neither of these correlators have the leading order contribution to the OPE, i.e.

$$
\langle 0|\rightarrow-\otimes \rightarrow \otimes \rightarrow-\otimes \rightarrow| 0\rangle=0 .
$$

Therefore, one is required to include the contributions to higher orders by means of diagrams with gluons coupled to the quark fields. Such diagrams naturally lead to contributions of the higher QCD condensates. The scheme below illustrates the contributions of the quark condensate $(D=3)$ in higher orders as well as the gluon condensate $(D=4)$, the quark-gluon condensate $(D=5)$, the four-quark condensate and the three-gluon condensate (both $D=6$ ) [21]:

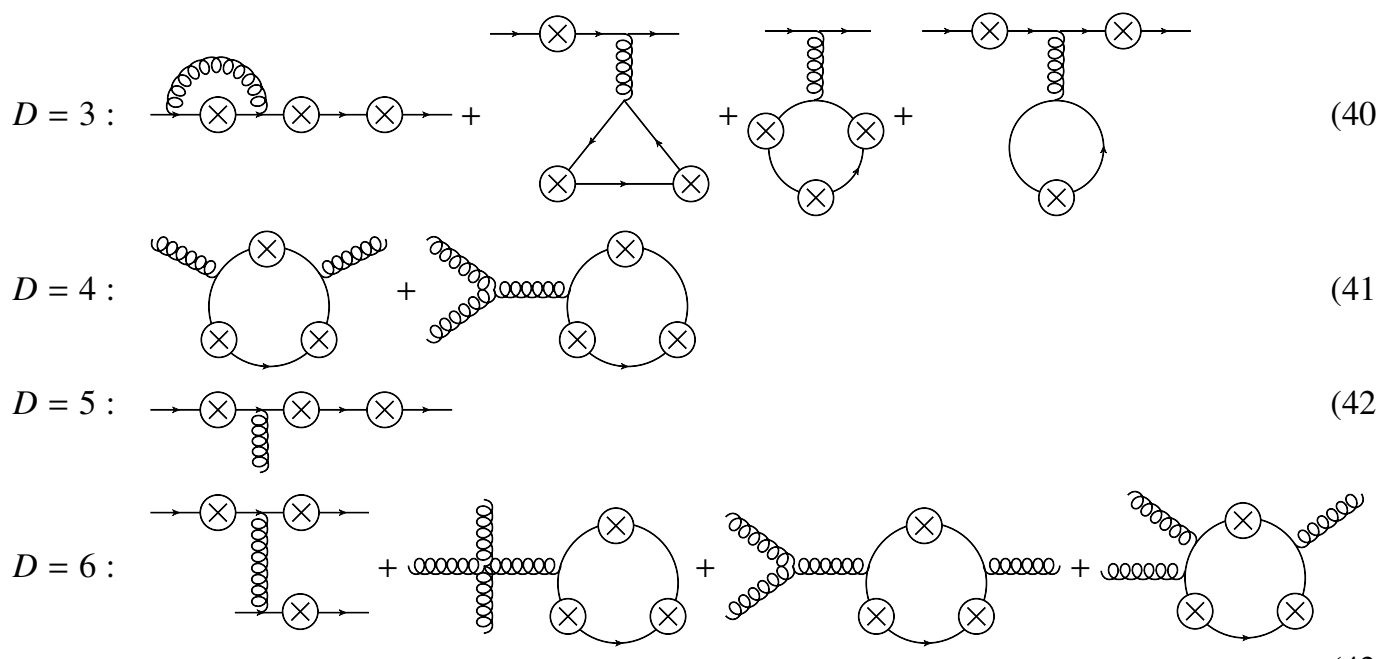

It is very important to mention that not all diagrams necessarily represent nontrivial contributions both for the $V V A$ and $A A A$ Green functions - some loops vanish due to the Furry theorem or due to a necessity to have the contributions Lorentz invariant. This study is now in progress [21].

\section{Conclusion}

In this article, we summarized the known results for the high-energy behaviour of Green functions within the OPE framework, relevant in the odd-intrinsic parity sector. We also presented our approach in order to evaluate the OPE for the $V V A$ and $A A A$ correlators for all momenta large.

\section{Acknowledgments}

Financial support by the Charles University (project GA UK no. 700214) and by the project GACR 15-18080S is gratefully acknowledged. 


\section{References}

[1] S. Weinberg, Physica A 96, 327-340 (1979)

[2] J. Gasser and H. Leutwyler, Annals Phys. 158, 142 (1984)

[3] J. Gasser and H. Leutwyler, Nucl. Phys. B 250, 465-516 (1985)

[4] G. Ecker, J. Gasser, A. Pich and E. de Rafael, Nucl. Phys. B 321, 311-342 (1989)

[5] B. Moussallam, Phys. Rev. D 51, 4939-4949 (1995)

[6] M. Jamin and V. Mateu, JHEP 0804, 040 (2008)

[7] M. A. Shifman, A.I. Vainshtein and Valentin I. Zakharov, Nucl. Phys. B 147, 448-518 (1979)

[8] M. A. Shifman, A.I. Vainshtein and Valentin I. Zakharov, Nucl. Phys. B 147, 385-447 (1979)

[9] K. Kampf and J. Novotný, Phys. Rev. D 84, 014036 (2011)

[10] M. Knecht, S. Peris, M. Perrottet and E. de Rafael, JHEP 0403, 035 (2004)

[11] T. Kadavý, K. Kampf and J. Novotný (in preparation)

[12] P. Colangelo, F. De Fazio, J. J. Sanz-Cillero, F. Giannuzzi and S. Nicotri, Phys. Rev. D 85, 035013 (2012)

[13] J. J. Sanz-Cillero, PoS QNP 2012, 118 (2012)

[14] G. P. Lepage and S. J. Brodsky, Phys. Rev. D 22, 2157 (1980)

[15] S. J. Brodsky and G. P. Lepage, Phys. Rev. D 24, 1808 (1981)

[16] B. Aubert et al. [BaBar Collaboration], Phys. Rev. D 80, (2009)

[17] S. Uehara et al. [Belle Collaboration], Phys. Rev. D 86, 092007 (2012)

[18] J. Gronberg et al. [CLEO Collaboration], Phys. Rev. D 57, 33-54 (1998)

[19] S. Peris, B. Phily and E. de Rafael, Phys. Rev. Lett. 86, 14-17 (2001)

[20] M. Knecht and A. Nyffeler, Eur. Phys. J. C 21, 659-678 (2001)

[21] T. Kadavý, K. Kampf and J. Novotný (in preparation) 